\title{
Camel milk modulates the gut microbiota and has anti-inflammatory effects in a mouse model of colitis
}

\author{
Jing He, ${ }^{1}$ Kunjie Guo, ${ }^{1}$ Qi Chen, ${ }^{1}$ Yulin Wang, ${ }^{1}$ and Jirimutu ${ }^{1,2 *}$ \\ ${ }^{1}$ Key Laboratory of Dairy Biotechnology and Engineering, Ministry of Education, College of Food Science and Engineering, \\ Inner Mongolia Agricultural University, Hohhot, Inner Mongolia, 010010, China \\ ${ }^{2}$ Camel Research Institute of Inner Mongolia, Alashan, Inner Mongolia, 750330, China
}

\begin{abstract}
Camel milk is a nutritionally rich food that shows anti-inflammatory, immune regulation, and gut microbiota maintenance properties. However, the relationship between camel milk and the intestinal microbiota during colitis is unclear. Herein, we evaluated the protective effect of camel milk in mice with colitis induced using dextran sodium sulfate. Our results showed that camel milk can prevent body weight loss and colon shortening, reduce the disease activity index, and attenuate colon tissue damage. Additionally, camel milk could reduce the overexpression of inflammatory factors, inhibit the apoptosis of intestinal epithelial cells, and promote the expression of claudin-1, occludin, and zonula occludens- 1 proteins. Moreover, camel milk effectively regulated intestinal microbiota in mice with colitis by increasing the gut microbiota diversity, increasing the abundance of beneficial bacteria (such as g_norank_f_Muribaculaceae, and Lachnospiraceae_NK4A136_group), and reducing the number of harmful bacteria (Bacteroides, Escherichia-Shigella). In addition, camel milk increased the levels of intestinal short-chain fatty acids. The results of the present study demonstrated that via regulating the intestinal microbiota, maintaining intestinal barrier function, and inhibiting proinflammatory cytokines, camel milk can ameliorate dextran sodium sulfate-induced colitis.
\end{abstract}

Key words: camel milk, gut microbiota, antiinflammatory, intestinal barrier function, dextran sodium sulfate

\section{INTRODUCTION}

Inflammatory bowel disease (IBD), which includes ulcerative colitis and Crohn's disease, comprises a chronic gastrointestinal tract inflammatory disease, which usually causes weight loss, abdominal pain, intes-

Received September 26, 2021.

Accepted January 13, 2022

*Corresponding author: yeluotuo1999@vip.163.com tinal bleeding, blood in the stool, and diarrhea (Chen et al., 2021). Recently, IBD incidence has risen rapidly and has become a global disease. Several factors have been implicated, including the function of the intestinal mucosal barrier, the environment, immunity, infection, genetics, and other factors; however, it remains unclear what causes IBD (Chen et al., 2008). Many studies have shown that an imbalance of the intestinal microbiota has an essential function in the development and occurrence of colitis, and in patients with IBD, the gut microbiota diversity differs markedly compared with that of healthy individuals (Gevers et al., 2014). Currently, the treatment of IBD relies mainly on drug therapy; however, these drugs are associated with side effects (Huang et al., 2019). Therefore, safe and effective prevention methods and drugs to alleviate IBD are urgently required. Recently, a diet-based strategy has been considered as a promising strategy to prevent IBD.

Recent studies have shown that patients with colitis are often associated with increased intestinal permeability, which is also closely related to inflammation (Bennebroek Evertsz' et al., 2012). Additionally, the gut microbiota has an essential function in the pathogenesis of IBD. An imbalance of the gut microbiota leads to epithelial cell damage and induces intestinal inflammation (Dinan and Cryan, 2017). The metabolites of gut microbes, including short-chain fatty acids (SCFA), effectively inhibit proinflammatory factor production by inhibiting the nuclear factor kappa B (NF-kB) pathway, thereby decreasing the occurrence of inflammatory reactions (Lavelle and Sokol, 2020). In addition, SCFA can reduce intestinal permeability and prevent colitis (Suzuki et al., 2008).

Camel milk is used as a natural health product mainly because of its remarkable antioxidation, immune regulation, anti-inflammatory, antidiabetic, and antiapoptotic properties (Ahamad et al., 2017; Aqib et al., 2019). Camel milk has a unique composition that differs from the milk of other ruminants. It contains higher levels of immunoglobulin, lactoferrin, and calcium, and lower levels of fat. Moreover, camel milk con- 
tains a variety of secreted antibodies, such as IgM and IgA, which have enhanced antiviral and antibacterial activities compared with cow milk. Additionally, camel milk comprises a variety of biologically active proteins with immunomodulatory effects, such as lactoferrin, lysozyme, lactoperoxidase, and $\mathrm{N}$-acetylglucosaminidase. The oligosaccharides in camel milk are not only conducive to the reproduction of intestinal bifidobacteria, but also inhibit the adhesion of pathogenic microorganisms to the colonic mucosa effectively. Recently, Cui et al. (2020) reported that camel milk effectively alleviated the damage to the colonic mucosa and the immune cell imbalance caused by dextran sodium sulfate (DSS) in mice. A separate study revealed that camel milk ameliorates 2,4,6-trinitrobenzene sulfonic acid-induced colitis via downregulation of inflammatory cytokines and oxidative stress (Arab et al., 2014). Therefore, in this study, we explored whether camel milk could alleviate enteritis by inhibiting inflammation, protecting the intestinal barrier, and improving the structure of the intestinal microbiome of mice with colitis. Our results provided evidence in favor of the use of camel milk as a dietary treatment of colitis.

\section{MATERIALS AND METHODS}

\section{Animals and Acute Colitis Induction}

Beijing Weitonglihua Laboratory Animal Technology Co. Ltd. (Beijing, China) provided male C57BL/6 mice (6-8 wk old). All mice were kept at $25 \pm 2^{\circ} \mathrm{C}$, at a relative humidity of $50 \pm 5 \%$, under a 12 -h light-dark cycle. As shown in Figure 1A, the mice were randomized into 4 groups ( $\mathrm{n}=8$ per group), including a control group (CK), a camel milk (CM) group $(10 \mathrm{~mL}$ of camel milk/kg), a $2.5 \%$ DSS (DSS) group, and a camel milk + DSS (CM+DSS) group (10 mL of camel milk $/ \mathrm{kg}$ and $2.5 \%$ DSS). After 1 wk of acclimation, CM was administered to the mice using oral gavage, and the mice were allowed free access the DSS solution $(2.5 \mathrm{~g}$ in 100 $\mathrm{mL}$ of drinking water) as their only water source from d 21 of the study. On the d 28, all the mice were killed. The animal experiments were performed according to the guidelines for the care and use of experimental animals of Inner Mongolia Agricultural University (ethics approval code: SYXK 2020-0002).

\section{Histological Evaluation and the Disease Activity Index}

Throughout the experiment, BW, blood in the stool, and stool consistency were monitored daily. The disease activity index (DAI) was evaluated using the scores of (1) bleeding (0: normal; 2: positive occult blood; 4: visible rectal bleeding), (2) diarrhea (0: normal; 1 and 2: loose stool; 3 and 4: diarrhea), (3) BW loss (0: none; 1: $1-5 \%$ loss; 2 : $5-10 \%$; and 3 : $10-20 \% ; 4:>20 \%$; Peng et al., 2019).

The colonic tissues were embedded, sectioned, and stained with hematoxylin and eosin $(\mathbf{H} \& \mathbf{E})$. The histopathological score was calculated according to the Cooper standard (Han et al., 2019). Tissue terminal deoxynucleotidyl transferase nick-end-labeling (TUNEL) staining was performed by section deparaffinization and hydration, cell permeabilization, addition of TUNEL reaction solution, addition of converter-peroxidase, color development with substrate 3,3'-diaminobenzidine, counting, and imaging under an optical microscope.

\section{Immunohistochemistry Analysis}

The sections were dewaxed, incubated in $3 \% \mathrm{H}_{2} \mathrm{O}_{2}$ to block endogenous peroxidase, and the antigen was repaired using citrate buffer. Then, nonspecific binding to the sections was blocked using goat serum at $37^{\circ} \mathrm{C}$ for $30 \mathrm{~min}$. The sections were then incubated overnight with primary antibodies [recognizing claudin-1, zonula occludens- 1 (ZO-1), and occludin] at $4^{\circ} \mathrm{C}$. The sections were rinsed thrice using PBS and then incubated with the secondary antibodies for $30 \mathrm{~min}$ at $37^{\circ} \mathrm{C}$. $3,3^{\prime}$-Diaminobenzidine was used to counter-stain the sections. The quantification of the positively stained areas for the 3 proteins was calculated using ImageJ (National Institutes of Health).

\section{ELISA Analysis}

Samples of mouse colon tissues were subjected to homogenization in PBS, followed by centrifugation at $14,000 \times g$ at $4^{\circ} \mathrm{C}$ for $15 \mathrm{~min}$. We then used ELISA to

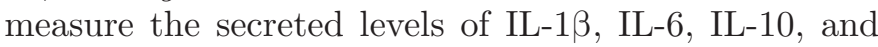
TNF- $\alpha$ in the supernatant.

\section{Gut Microbiota Analysis}

Total bacterial DNA was extracted from the contents of the mouse colon using a DNA Extraction Kit (Qiagen Inc.) following the manufacturer's instructions. The DNA was quality checked and quantified using agarose gel electrophoresis and a NanoDrop spectrophotometer (Nanodrop Technologies), respectively. We used PCR to amplify the $16 \mathrm{~S}$ rRNA gene V3-V4 regions using forward primer 338F 5'-ACTCCTACGGGAGGCAGCA-3' and reverse primer 806R 5'-GGACTACHVGGGTWTCTAAT- $3^{\prime}$. The PCR products were further purified using an AxyPrepDNA Gel Recovery Kit (Axygen Biosciences). Then, sequencing was performed using the Illumina Miseq PE300 system (Illumina) to 
A
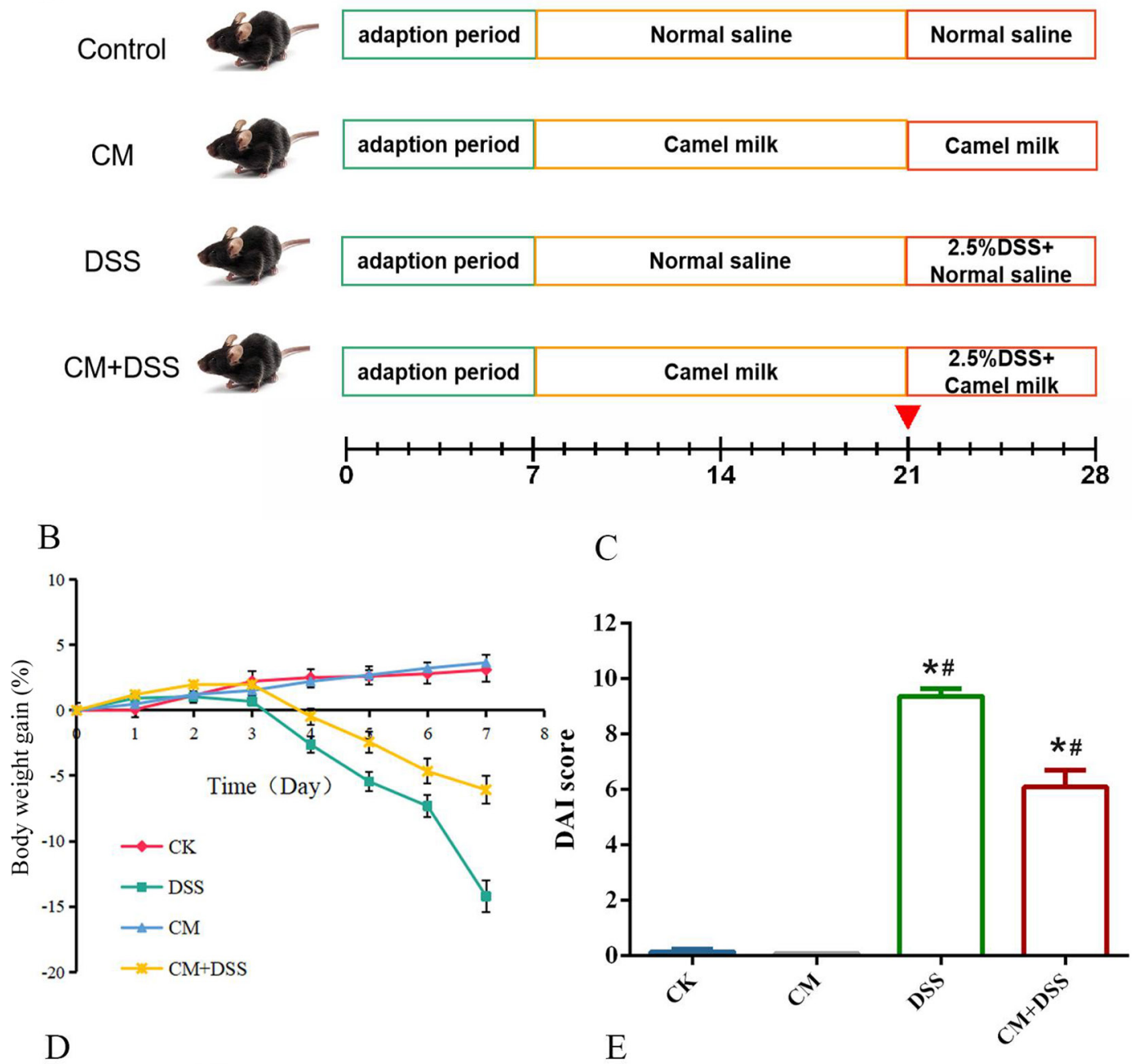

$\mathrm{D}$
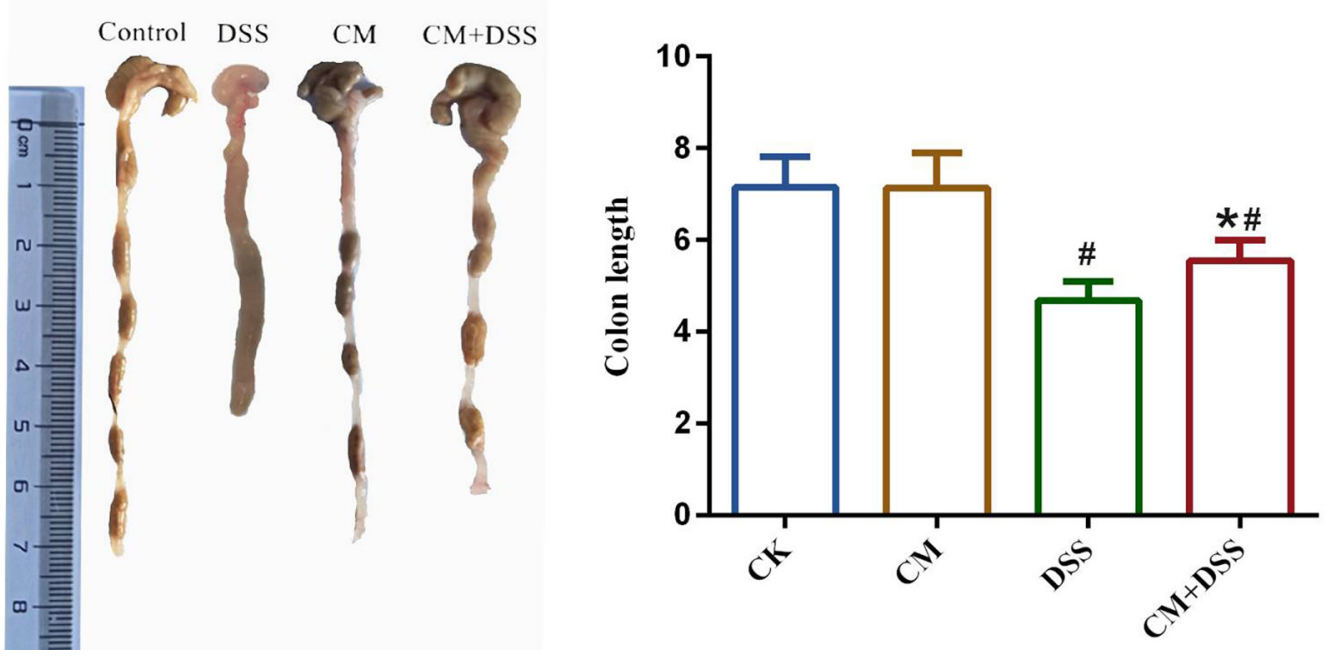

Figure 1. Effects of camel milk on the symptoms of colitis induced by dextran sodium sulfate (DSS) in mice. (A) Experimental protocol; (B) changes in BW; (C) disease activity index (DAI); (D) images of the lengths of the colon; (E) graph of colon lengths (cm). Data in panels B, $\mathrm{C}$, and $\mathrm{D}$ are shown as mean $\pm \mathrm{SD} .{ }^{*} P<0.05$ versus the DSS group; $\# P<0.05$ versus the CK group. $\mathrm{CM}=$ camel milk group; CK $=$ control group; CM+DSS = camel milk with DSS. 
sequence the PCR amplicons. The operational taxonomic units were clustered using Usearch (version 7.0) with a similarity of $97 \%$. We used QIIME-generated (http://qiime.org/) operational taxonomic units to investigate the $\alpha$ and $\beta$ diversities. $\alpha$ Diversity indices (Chao1 and Shannon) were measured with Mothur (version v.1.30.1). The $\beta$ diversity was calculated using Bray-Curtis with principal coordinate analysis and nonmetric multidimensional scaling to illustrate the similarities and differences among the microbial communities. Cladogram construction and linear discriminant analysis were performed using the LEfSe (linear discriminant analysis effect size) tool.

\section{Analysis of SCFA}

One hundred milligrams of mouse cecum content was combined with $1 \mathrm{~mL}$ of $0.005 M \mathrm{NaOH}$, vortexed for $10 \mathrm{~min}$, and centrifuged at 13,200 $\times g$ for $20 \mathrm{~min}$ at $4^{\circ} \mathrm{C}$. The SCFA were identified by comparison with the chemical standards (acetic acid, propionate acid, and butyric acid), and caproic acid-d3 was used as the internal reference. The organic phase was filtered into a tube and subjected to GC-MS.

\section{Data Analysis}

One-way parametric analysis (ANOVA) followed by Tukey's post hoc test was used to analyze the significance of differences between the groups in GraphPad 7.5 software (GraphPad Inc.). Data are displayed as the mean \pm standard deviation. $P<0.05$ was considered a significant difference.

\section{RESULTS AND DISCUSSION}

\section{Camel Milk Alleviated the Symptoms of DSS-Induced Colitis in Mice}

Induction of colitis in mice using DSS is a well-established preclinical model, which has a histopathology similar to that of human IBD (Sun et al., 2020). As shown in Figure 1B, during the entire experiment, the BW of mice in the CK and CM groups increased continuously. The mice in the DSS and CM+DSS groups exhibited notable weight loss; however, the CM+DSS group experienced less weight loss that the DSS group. The DAI score is comprehensively evaluated by combining stool consistency, fecal blood, and BW loss. As shown in Figure 1C, the DAI score for the DSS group was much higher than that for the CK group, and the addition of CM effectively decreased the DAI score $(P$ $<0.05)$. The DSS induces histological changes that results in colon shortening. The shortening of the colon is a marker of the severity of inflammation in colitis (Chen et al., 2021). The results demonstrated that that $\mathrm{CM}$ reduced colon shortening in mice with colitis (Figure $1 \mathrm{D}$ and $1 \mathrm{E} ; P<0.05)$. These data suggested that $\mathrm{CM}$ ameliorated colitis progression indicators, which is consistent with previous researches (Arab et al., 2014; Cui et al., 2020).

\section{Camel Milk Ameliorated Colonic Tissue Damage in Mice}

Figure 2A shows the histological changes in the colons of mice as revealed using H\&E staining. The CK group colonic tissues showed normal morphology without inflammation. No significant differences were found between the CK and CM groups. In contrast, the DSS group exhibited disruption of crypts, severe epithelial erosion, loss of goblet cells, and inflammatory cell infiltration; consequently, their histopathological scores were much higher than those of the CK group (Figure $2 \mathrm{C} ; P<0.05$ ). However, $\mathrm{CM}$ supplementation reduced the morphological alterations and protected the colonic tissue integrity $(P<0.05)$. The number of TUNEL-positive cells in the tissues was counted to estimate colonic cell apoptosis (Figure 2B). The colonic epithelial tissues of the CK group and the CM group showed no apoptosis characteristics, whereas DSS-induced colitis caused a dramatic increase in the number of TUNEL-positive cells $(P<0.05)$. However, the colonic epithelium of the CM+DSS group had a significantly decreased number of apoptotic cells compared with that of the DSS only-treated mice (Figure $2 \mathrm{D} ; P<0.05)$.

\section{Camel Milk Inhibited the Production of Excess Inflammatory Cytokines}

Inflammatory cytokines have crucial roles in the inflammatory response, and their levels can indicate the extent of inflammation (Liang et al., 2018). As shown in Figure 3, the secretory levels of TNF- $\alpha$, IL6 , IL-10, and IL-1 $\beta$ in the DSS group were increased significantly $(P<0.05)$. However, in the $\mathrm{CM}+\mathrm{DSS}$ group, oral consumption of $\mathrm{CM}$ decreased the levels of secretory inflammatory cytokines significantly compared with those in the DSS group $(P<0.05)$. In the pathogenesis of IBD, proinflammatory cytokines are important, as they are responsible for regulating the immune response and intestinal inflammation responses (Du et al., 2018). Macrophages and monocytes mainly secrete TNF- $\alpha$ and IL-1 $\beta$, and macrophage activation can promote the proinflammatory response (Du et al., 2018). In particular, TNF- $\alpha$ is essential for the persistence of DSS-induced colitis, and the increase of 
A
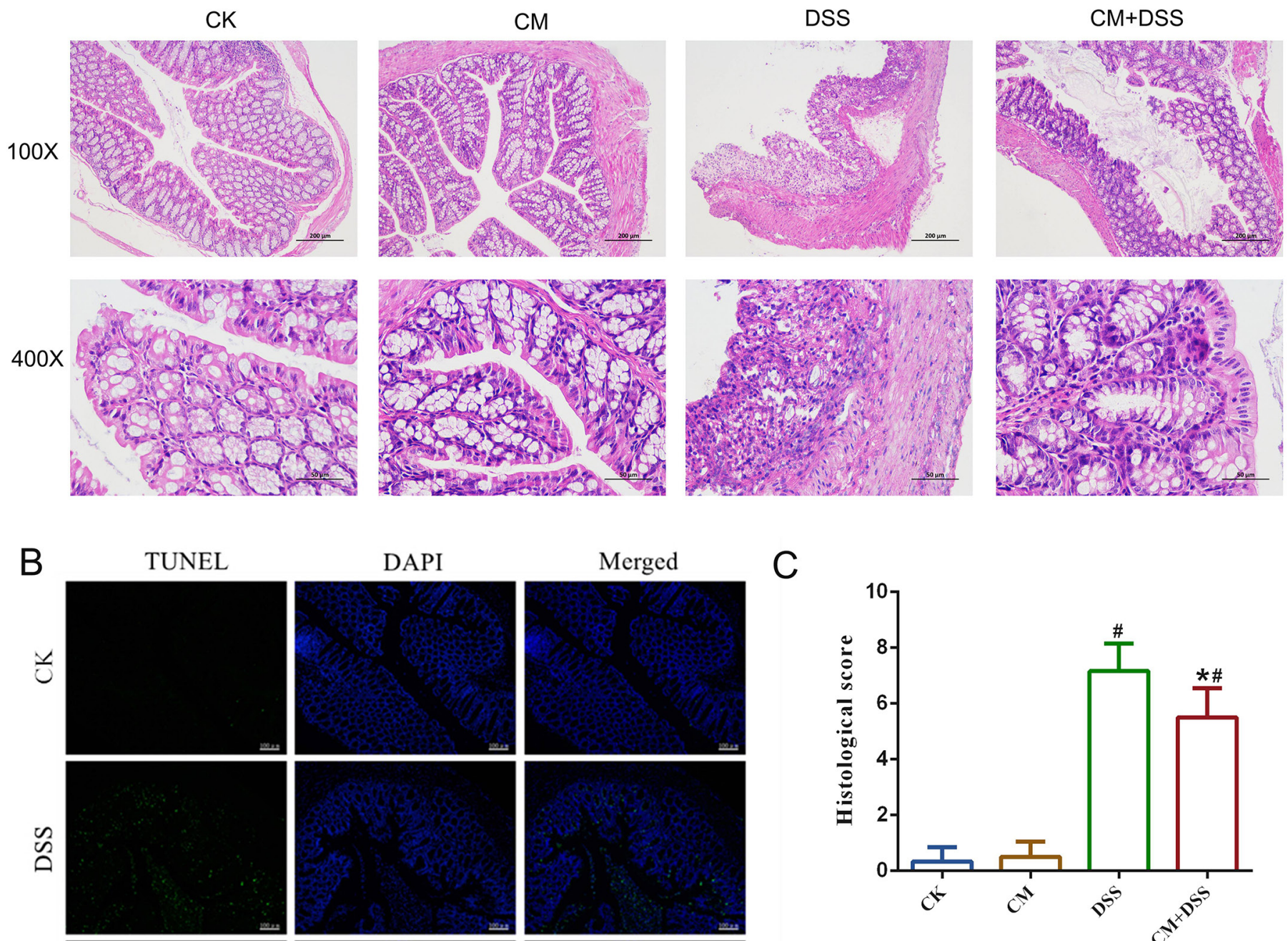

C
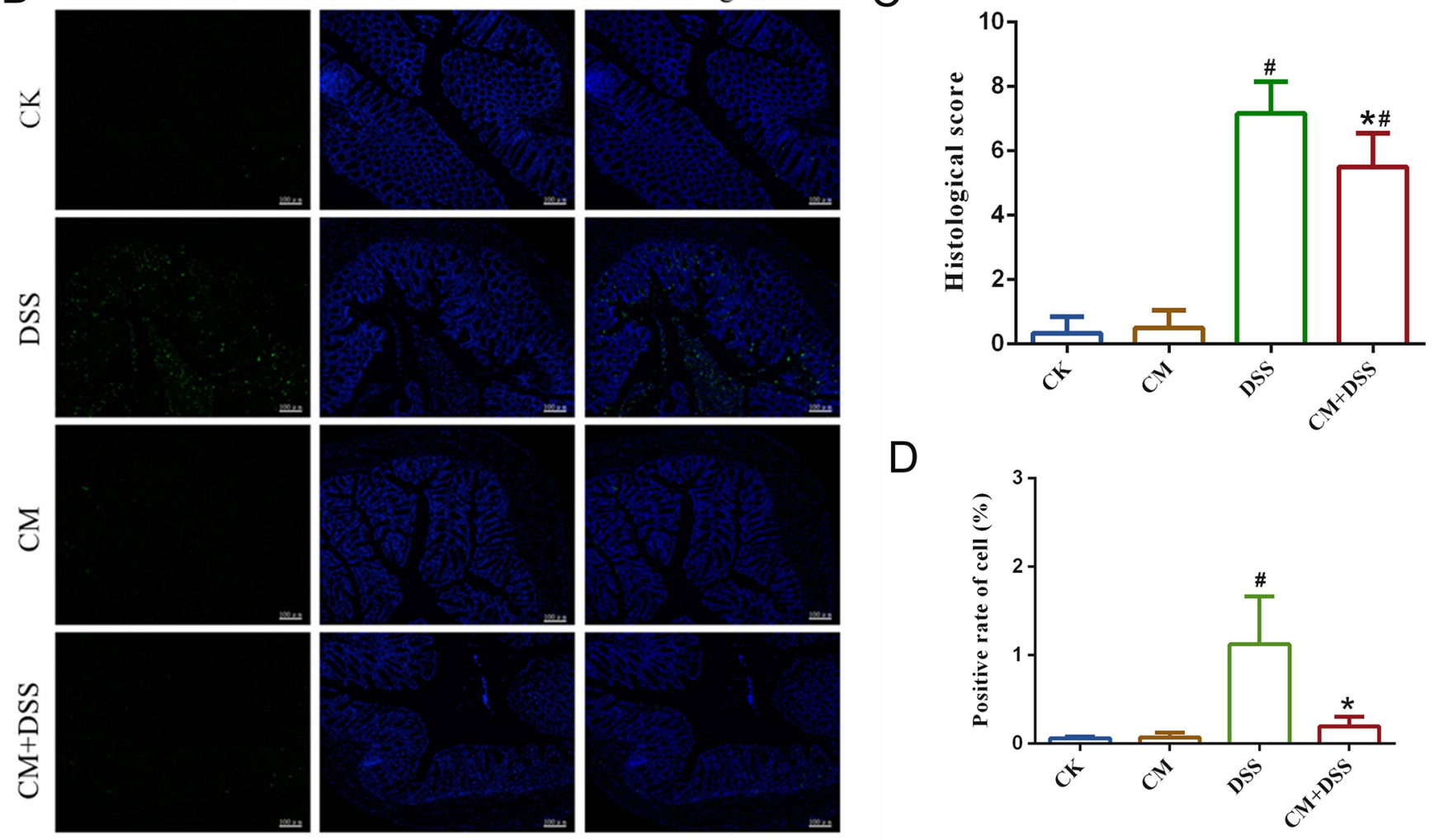

Figure 2. Effect of camel milk on dextran sodium sulfate (DSS)-induced damage to the colon. (A) Representative images of distal colon tissues stained with hematoxylin and eosin (100× and 400× magnification); (B) representative images of colon cell apoptosis with TUNEL (terminal deoxynucleotidyl transferase nick-end-labeling) and DAPI (4',6-diamidino-2-phenylindole) staining; (C) histological scores; (D) semiquantitative determination of the number of TUNEL-positive cells. Data are shown as the mean \pm SD; ${ }^{*} P<0.05$ versus the DSS group; \#P< 0.05 versus the CK group. $\mathrm{CM}=$ camel milk group; $\mathrm{CK}=$ control group; $\mathrm{CM}+\mathrm{DSS}=$ camel milk with DSS. 
TNF- $\alpha$ also upregulates IL-1 $\beta$ and IL- 6 secretion (Chen et al., 2021). Our results showed that the production of IL-1 $\beta$, IL-6, and TNF- $\alpha$ in the colon was significantly inhibited in mice with colitis administered with CM $(P$ $<0.05)$. IL-10 reduces secretion of proinflammatory cytokines (IL-1 $\beta$ and TNF- $\alpha$ ), thus restraining inflammation (Shu et al., 2014). Interestingly, the result showed that DSS treatment increased IL-10 levels significantly. This might reflect a self-defense mechanism in response to the more severe colitis induced by DSS (Pei et al., 2019). Supplementation of CM effectively decreased the level of the 4 cytokines in the CM+DSS group. In light of these results, we hypothesized $\mathrm{CM}$ can at least par- tially reduce the severity of acute colitis via reducing the levels of proinflammatory cytokines.

\section{Camel Milk Restored Gut Barrier Integrity in DSS-Treated Mice}

Figure 4 shows the immunohistochemistry results for claudin-1, occludin, and ZO-1. The levels of these 3 proteins decreased significantly after treatment with DSS compared with those in the CK group $(P<0.05)$, whereas supplementation with $\mathrm{CM}$ increased their levels significantly $(P<0.05)$. A crucial effector leading to IBD is the destruction of the intestinal epithelial
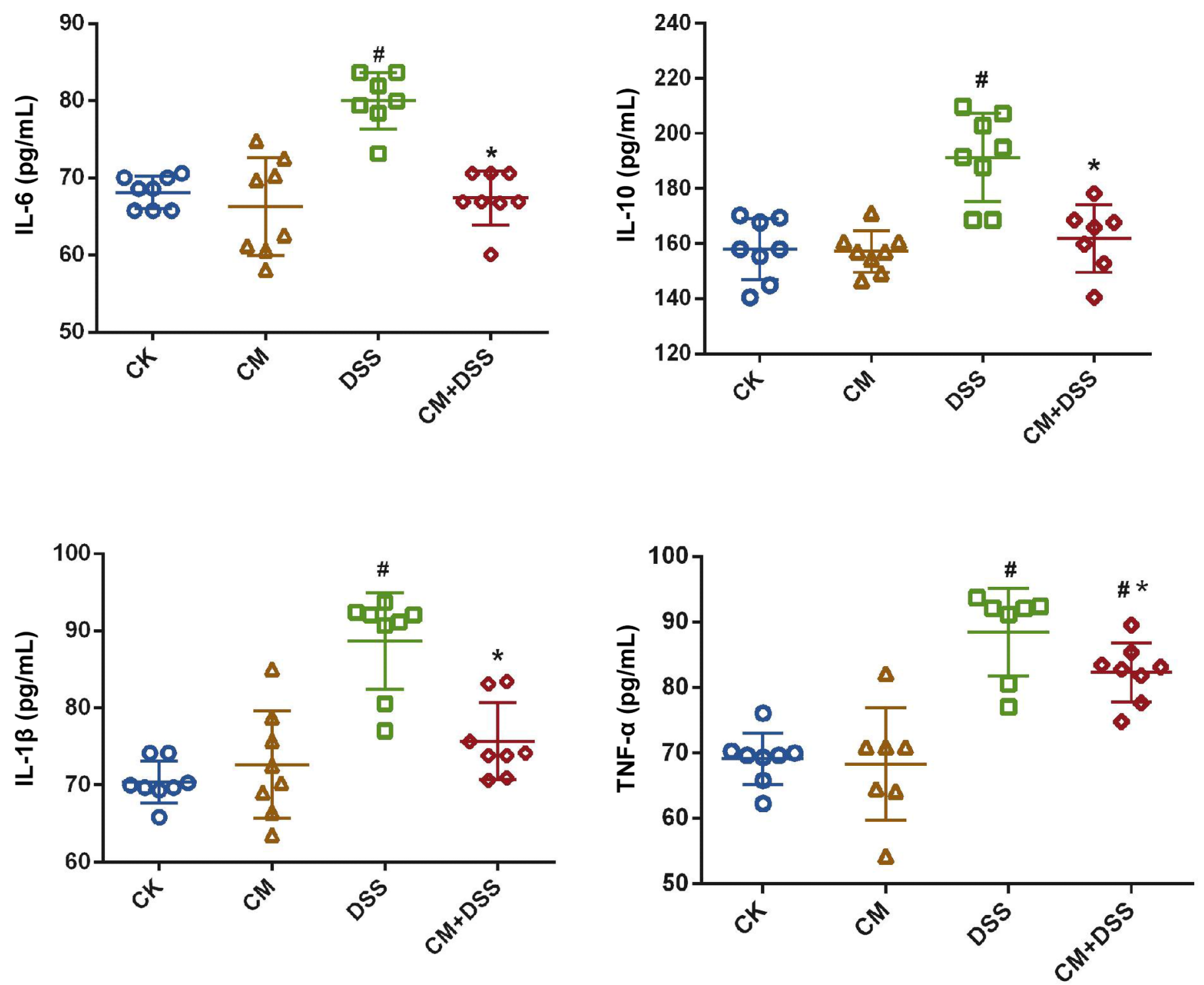

Figure 3. Effect of camel milk on inflammatory cytokine levels (IL-6, IL-10, IL-1 $\beta$, and TNF- $\alpha$ ) by ELISA in colonic tissues. Data are shown as the mean $\pm \mathrm{SD} ;{ }^{*} P<0.05$ versus the dextran sodium sulfate (DSS) group; $\# P<0.05$ versus the CK group. CM $=$ camel milk group; CK $=$ control group; CM+DSS $=$ camel milk with DSS. 
A
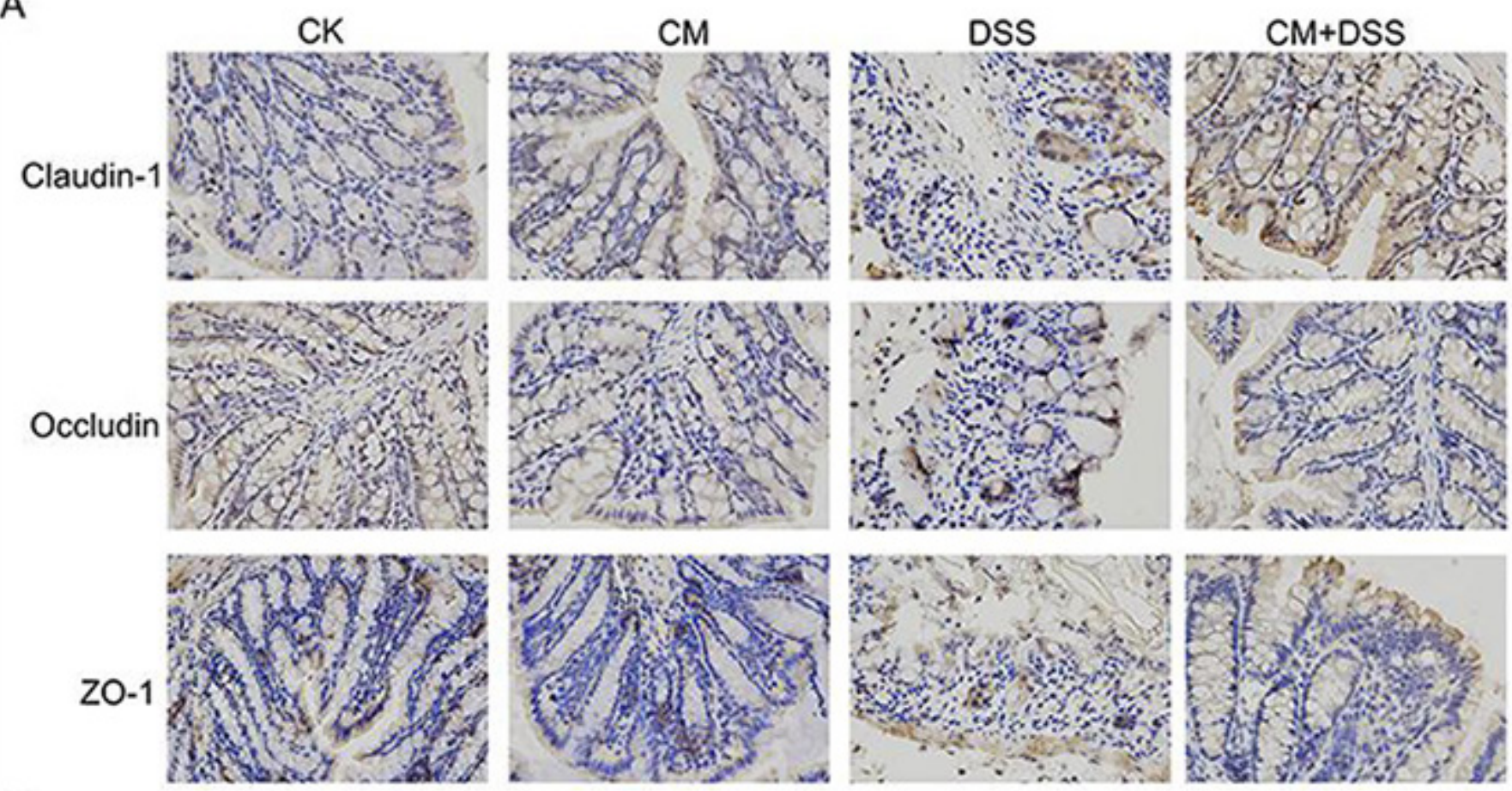

B
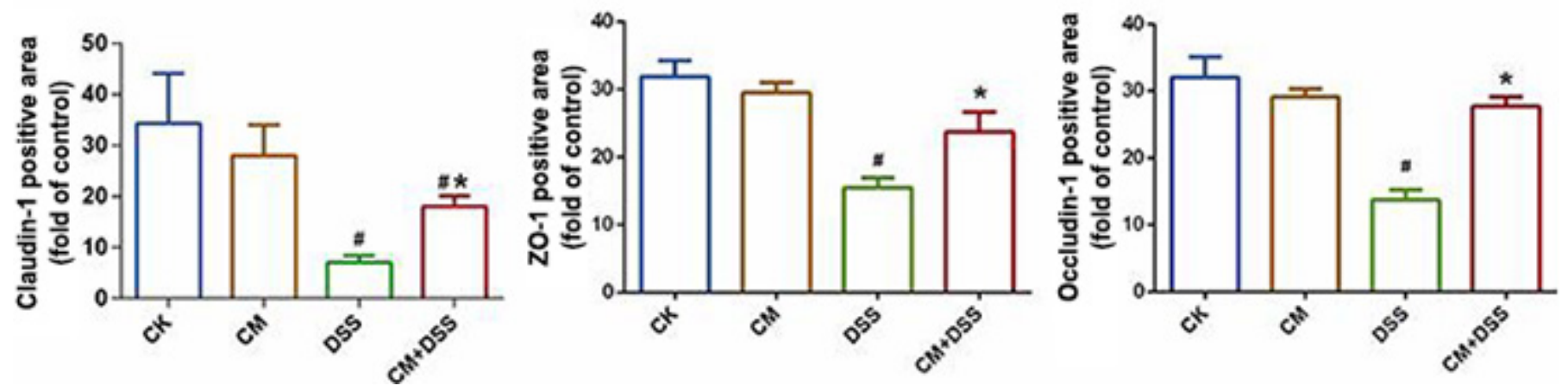

Figure 4. The effects of camel milk on mouse gut barrier integrity in colonic tissues. (A) Representative photographs of the expression of claudin-1, occludin, and ZO-1 (magnification 400×); (B) quantification of positively stained areas for claudin-1, occludin, and ZO-1 calculated using ImageJ (National Institutes of Health). Data are shown as the mean $\pm \mathrm{SD} ;{ }^{*} P<0.05$ versus the dextran sodium sulfate (DSS) group; \#P $<0.05$ versus the $\mathrm{CK}$ group. $\mathrm{CM}=$ camel milk group; $\mathrm{CK}=$ control group; $\mathrm{CM}+\mathrm{DSS}=$ camel milk with DSS.

barrier. Once the permeability of the epithelium increases, pathogenic bacteria and antigens will invade cells, which activates the host's innate immune system (Zeng et al., 2017). An excessive immune response and the overproduction of inflammatory cytokines harm the mucosal barrier of the intestine, which aggravates the abnormal mucosal immune response in patients with IBD (Schoultz and Keita, 2019). Therefore, to treat IBD, the integrity of the intestinal epithelial barrier should be maintained. Impaired epithelial function is associated with claudin-1, occludin, and ZO-1 (Su et al., 2009, Lin et al., 2019). ZO-1, a major tight junctions protein, is related to the integrity of the intestinal epithelium and is used as a marker for the intestinal mechanical barrier (Zhang et al., 2021). Occludin is vital for the stability of tight junctions and barrier function (Zhang et al., 2021). The membrane protein claudin-1 is a tight junction component (Peng et al., 2019). Thus, these data indicated that $\mathrm{CM}$ could prevent colitis in mice by improving intestinal barrier integrity.

\section{Camel Milk Partially Ameliorated Gut Microbiota Dysbiosis}

Accumulating evidence suggests that the gut microbiota is a critical factor in the pathogenesis of IBD 
A
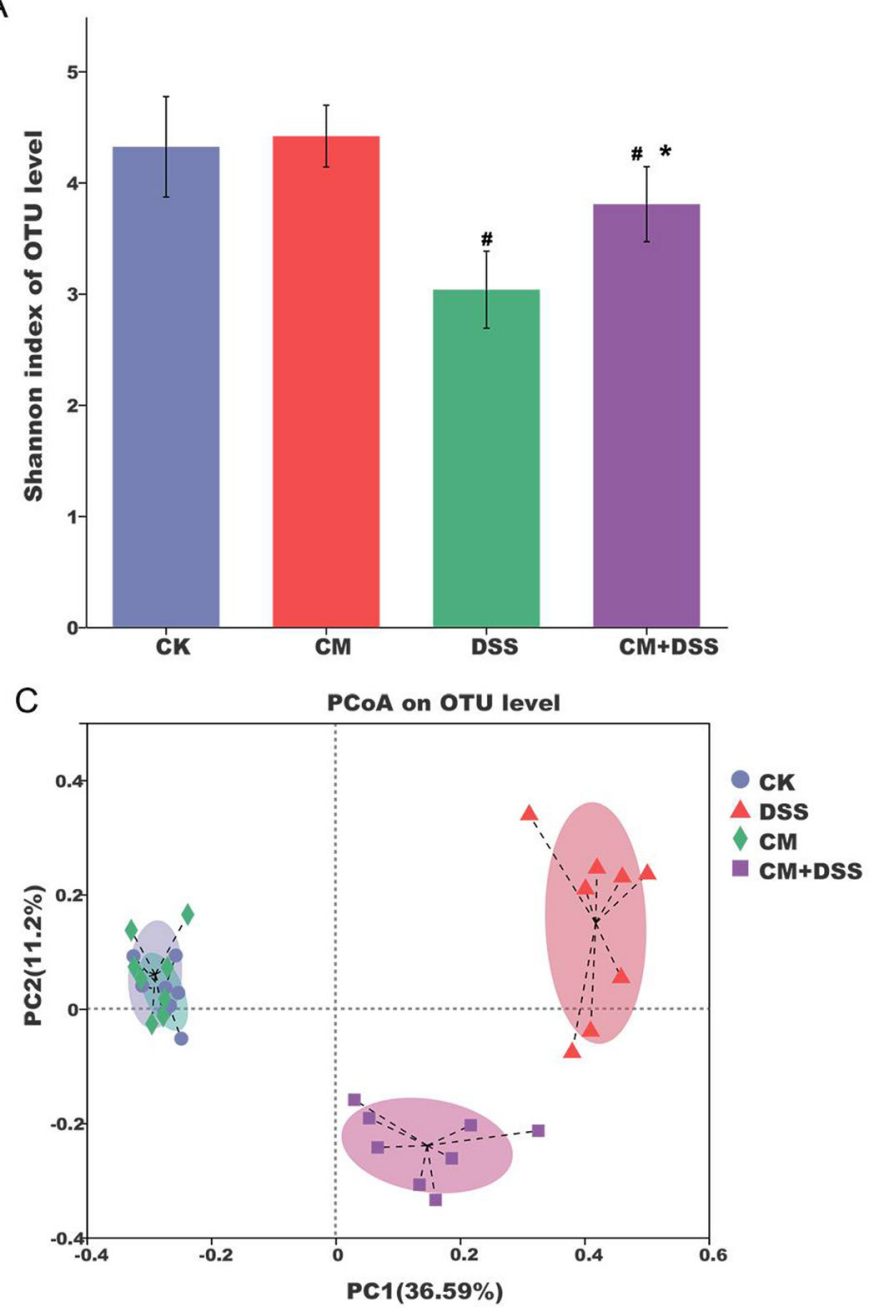

B
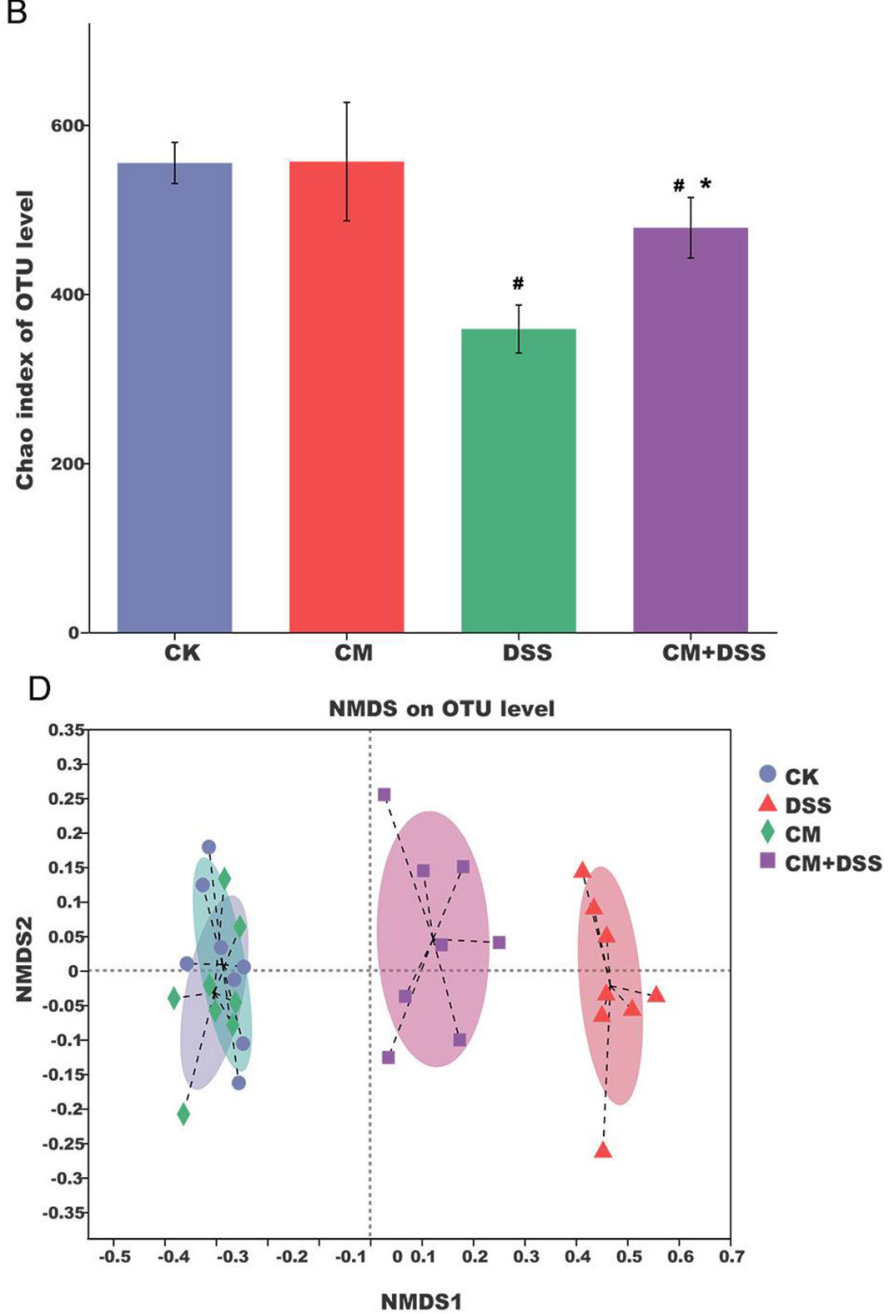

Figure 5. The effects of camel milk on colonic microbiota diversity. (A) Comparison of the Shannon index-derived $\alpha$-diversities. (B) Comparison of the Chao index-derived $\alpha$-diversities. (C) Gut microbiota communities' principal coordinate analysis based on weighted UniFrac distances (D) nonmetric multidimensional scaling (NMDS) of gut microbiota communities by Bray-Curtis. Data are shown as the mean \pm SD; ${ }^{*} P<0.05$ versus the dextran sodium sulfate (DSS) group; $\# P<0.05$ versus the CK group. $\mathrm{CM}=$ camel milk group; CK $=$ control group; $\mathrm{CM}+\mathrm{DSS}=$ camel milk with DSS; OTU $=$ operational taxonomic units.

(Frank et al., 2007). Alteration in the microbial community diversity and structure of patients with IBD was associated with colonic inflammation. To evaluate the microbial diversity affected by camel milk, an $\alpha$-diversity analysis was performed. Supplementation with CM increased the Chao and Shannon indices significantly compared with those in the DSS group (Figure $5 \mathrm{~A}, \mathrm{~B} ; P<0.05)$. Then, the similarity between the microbial communities was measured using $\beta$-diversity analysis (principal coordinate analysis and nonmetric multidimensional scaling; Figure 5C, D). The results showed that, compared with the CK and CM groups, DSS treatment significantly altered the gut microbiota. Moreover, CM supplementation shifted this grouping back toward the CK group, which is clearly differ- ent from the DSS group. Figure 6 shows the LEfSe analysis of the changes in the gut microbiota among the groups. Figure $6 \mathrm{~B}$ shows marked differences in different groups. We observed that DSS increased the level of Bacteroides, Escherichia-Shigella, Parabacteroides, and Klebsiella in the gut. However, the levels of Machnospiraceae_NK4A136, Odoribacter, Romboutsia, and Prevotellaceae-UCG-001 in the CM+DSS group were dominant in the microbiota.

Next, we analyzed the gut microbial changes among the groups at the family and genus levels to reveal the effect of CM on the gut microbial structure of mice with colitis and healthy mice. At the family level, the gut microbiota comprised Lachnospiraceae, Muribaculaceae, Bacteroidaceae, and Prevotellaceae in our experimental 
A

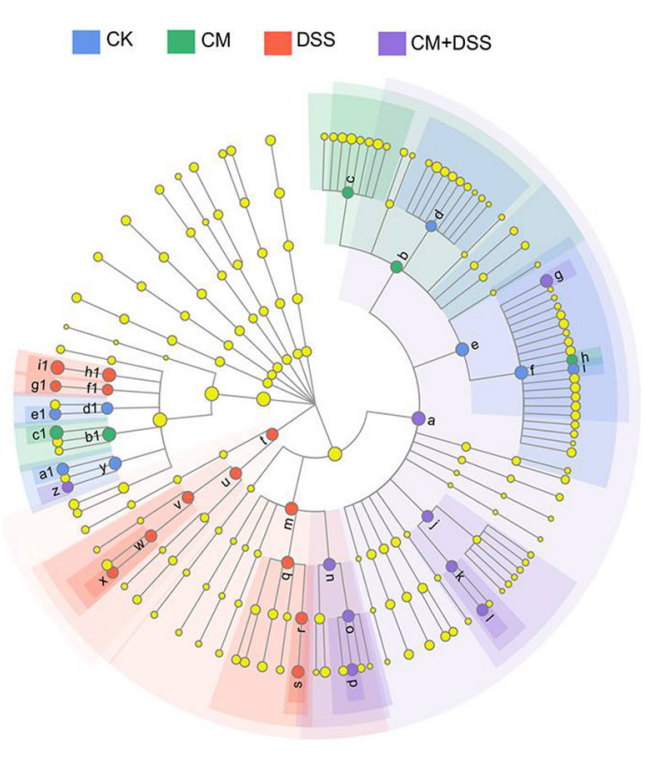

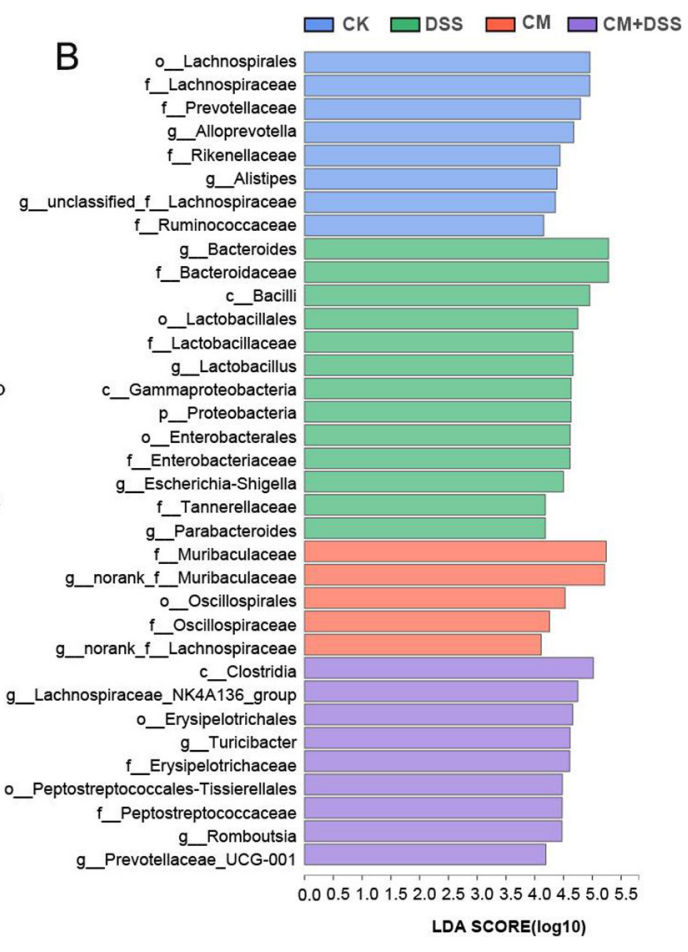

Figure 6. The colonic microbiota analyzed using LEfSe. (A) LEfSe analysis-derived taxonomic cladogram. (B) Linear discriminant analysis scores of the differentially abundant taxa. $\mathrm{CM}=$ camel milk group; $\mathrm{CK}=$ control group; DSS $=$ dextran sodium sulfate; CM+DSS = camel milk with DSS.

mice (Figure 7A). After DSS treatment, Bacteroidaceae and Enterobacteriaceae levels increased markedly, whereas the relative abundance of Lachnospiraceae and Muribaculaceae were decreased significantly compared with those in the CK group (Figure 7B). Enterobacteriaceae comprise opportunistic pathogens, which might upregulate inflammatory cytokine production and induce innate immunity-related cells (Jacobson et al., 2018). In addition, the increased abundance of Bacteroidaceae in colitis also promotes immunosuppression and carcinogenesis (Ryan et al., 2020). However, dietary CM reversed the decreases in Lachnospiraceae and Muribaculaceae abundance induced by DSS (Figure 7B). Lachnospiraceae are intestinal bacteria that produce SCFA such as butyric acid (Peng et al., 2020). Muribaculaceae are positively related to the barrier function of the intestinal mucus layer and play a role in the degradation of complex carbohydrates (Lagkouvardos et al., 2019).

At genus level, as shown in the Figure $8 \mathrm{C}$, norank_f_Muribaculaceae, Bacteroides, Lachnospiraceae_NK4A136, and Alloprevotella showed relatively high abundance. Compared with those in the CK group, the levels of Bacteroides and Escherichia-Shigella increased significantly in the DSS group $(P<0.05$; Figure $7 \mathrm{D})$. Colitis in mice can be induced by commensal Bacteroi- des, which supports their potential proinflammatory effects in mice with colitis (Bloom et al., 2011), as reflected by their higher abundance in the DSS group compared with that in the CK group in this study. In addition, Escherichia-Shigella is a typical pathogenic bacterium that can promote the physiological changes of the intestinal epithelium and tissue invasion by secreting specific virulence factors (Peng et al., 2020). They might participate in the destruction of the tight junctions of the intestinal mucosal barrier. However, after CM intervention, not only were the levels of harmful bacteria (including Bacteroides and Escherichia-Shigella) lower than those in the DSS group, but the levels of the beneficial bacteria (such as norank_f_Muribaculaceae and Lachnospiraceae_NK4A136) were higher than those in the DSS group. Lachnospiraceae_NK4A136_group, the principal genus of Lachnospiraceae, correlated positively with the pathological features of colitis (Wang et al., 2019). Muribaculaceae, the primary gut microbiota found in healthy individuals, can produce acetic acid and propionic acid (Shao et al., 2020). Additionally, compared with those in the DSS group, the levels of SCFA-producing bacteria (Odoribacter, Romboutsia, and Prevotellaceae-UCG-001) were higher in the $\mathrm{CM}+\mathrm{DSS}$ group, which would improve the intestinal barrier function, thereby reducing inflammation (Shi et 


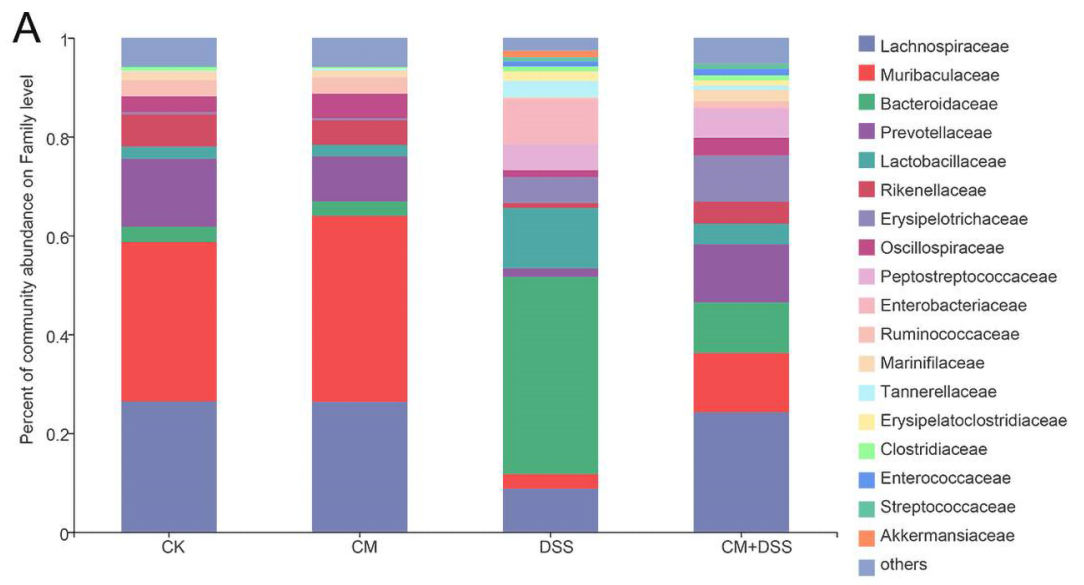

B
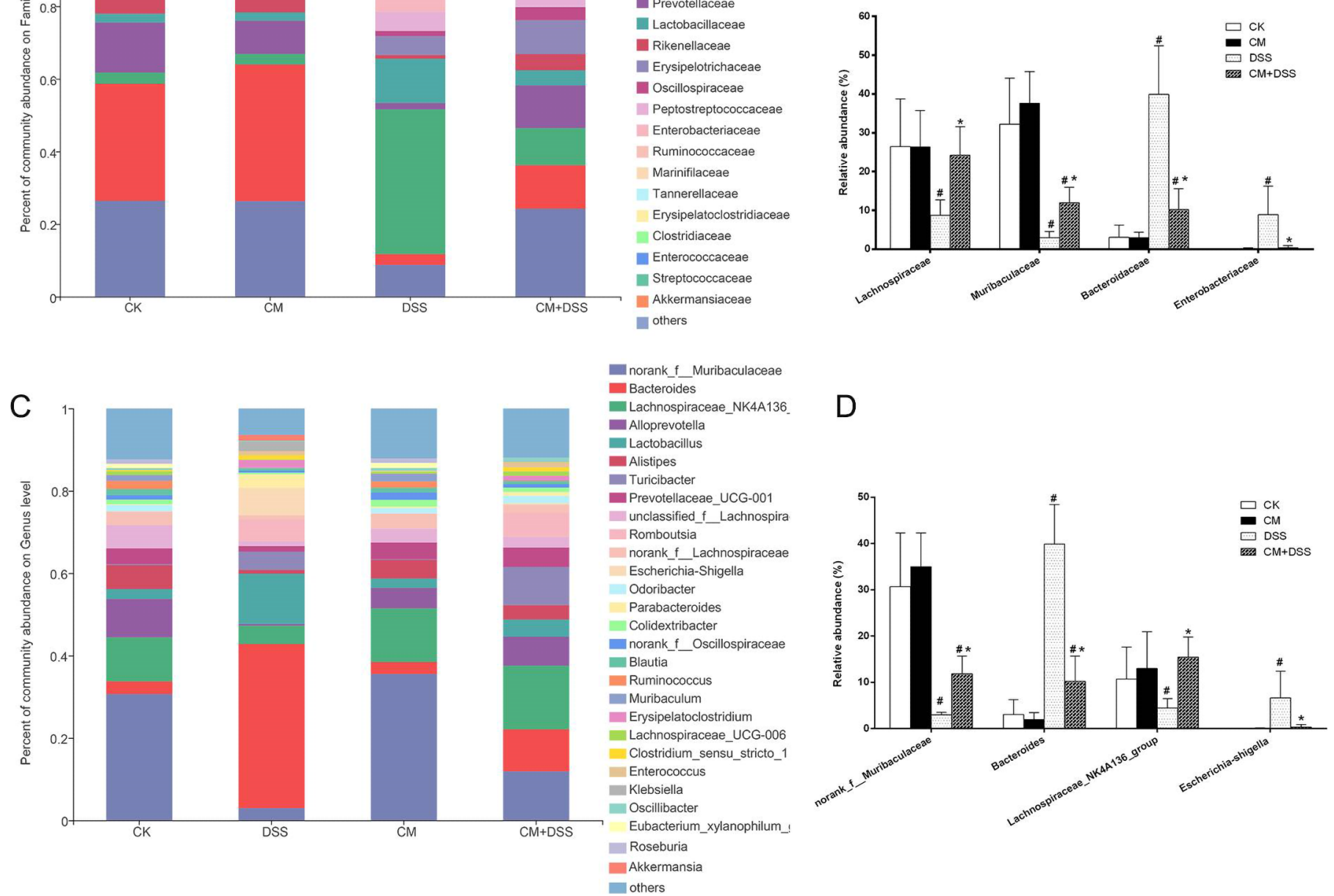

Figure 7. The effects of camel milk on the composition of the colonic microbiome in mice treated with DSS. (A) The gut microbiota relative abundance at the family level. (B) Family level relative differences in the gut microbiota in each group. (C) The gut microbiota relative abundance at the genus level. (D) Genus level relative differences in the gut microbiota in each group. Data are shown as the mean $\pm \mathrm{SD} * P$ $<0.05$ versus the DSS group; \#P $<0.05$ versus the CK group. $\mathrm{CM}=$ camel milk group; $\mathrm{CK}=$ control group; DSS $=$ dextran sodium sulfate; $\mathrm{CM}+\mathrm{DSS}=$ camel milk with DSS.

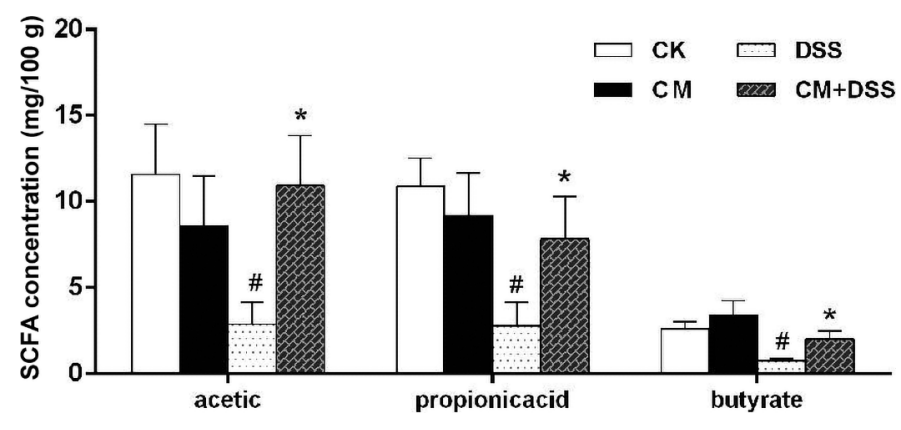

Figure 8. Effects of camel milk on the production of short-chain fatty acids (SCFA) in cecum of colitic mice. Data are shown as the mean $\pm \mathrm{SD}$. ${ }^{*} P<0.05$ versus the dextran sodium sulfate (DSS) group; $\# P<0.05$ versus the $\mathrm{CK}$ group. $\mathrm{CM}=$ camel milk group; $\mathrm{CK}=$ control group; DSS $=$ dextran sodium sulfate; $\mathrm{CM}+\mathrm{DSS}=$ camel milk with DSS. al., 2021). Taken together, our data shows that CM has a potential beneficial effect by promoting the growth of specific probiotics by beneficial intestinal bacteria.

\section{Camel Milk Recovered the Production of SCFA Induced by DSS}

Short-chain fatty acids are produced by the gut microbiota and are critical to maintain human intestinal health. Changes in intestinal flora, which lead to changes in SCFA, correlate strongly with colitis (Maslowski et al., 2009). As shown in Figure 8, the acetate, propionate, and butyrate contents in the DSS group were significantly lower than those in the CK group $(P<0.05)$. Compared with those in the DSS 
treatment group, the acetate, propionate, and butyrate levels of mice pretreated with CM increased significantly. Studies have proposed that SCFA produced by microbial fermentation can be used to treat IBD (Vinolo et al., 2011). Short-chain fatty acids, particularly propionic acid and butyric acid, have been reported to protect the intestinal mucosa and suppress inflammatory cytokines production (Peng et al., 2019). Based on these observations, we speculated that camel milk's anti-inflammatory effect might be partly caused by enhancement of gut SCFA production.

\section{CONCLUSIONS}

The results showed that camel milk could inhibit the inflammatory response by suppressing the overexpression of inflammatory cytokines in the colon. In addition, camel milk increased the expression of ZO-1, occludin, and claudin- 1 to restore the function of the intestinal barrier function. Finally, camel milk regulated the intestinal microbiota of the mice with colitis by enhancing gut microbiota diversity, altering the abundance of the gut microbiota, and enhancing the SCFA concentration. Overall, camel milk was not only effective in decreasing DSS-treated colonic inflammation, but also regulated the structure of the intestinal microbiota. The results of the present study suggest that camel milk can be used to prevent colonic inflammation.

\section{ACKNOWLEDGMENTS}

This work was supported by the Natural Science Foundation of Inner Mongolia Autonomous Region, Hohhot, China (2020BS03040) and the Inner Mongolia Agricultural University high-level talent introduction scientific research start-up project, Hohhot, China (NDYB2019-26). Jing He and Kunjie Guo accomplished most of the experiments. Jing He and Kunjie Guo analyzed the results. Jing He wrote the manuscript. Chen Qi and Yulin Wang helped with the experiments. Jing He and Jirimutu designed this study and modified the manuscript. All authors listed have approved it for publication. The authors have not stated any conflicts of interest.

\section{REFERENCES}

Ahamad, S. R., M. Raish, A. Ahmad, and F. Shakeel. 2017. Potential health benefits and metabolomics of camel milk by GC-MS and ICP-MS. Biol. Trace Elem. Res. 175:322-330.

Aqib, A. I., M. Fakhar-e-Alam Kulyar, K. Ashfaq, Z. A. Bhutta, M. Shoaib, and R. Ahmed. 2019. Camel milk insuline: Pathophysiological and molecular repository. Trends Food Sci. Technol. 88:497-504. https://doi.org/10.1016/j.tifs.2019.04.009.
Arab, H. H., S. A. Salama, A. H. Eid, H. A. Omar, E.-S. A. Arafa, and I. A. Maghrabi. 2014. Camel's milk ameliorates TNBS-induced colitis in rats via downregulation of inflammatory cytokines and oxidative stress. Food Chem. Toxicol. 69:294-302. https://doi.org/ 10.1016/j.fct.2014.04.032.

Bennebroek Evertsz', F., N. Thijssens, P. Stokkers, M. Grootenhuis, C. Bockting, P. Nieuwkerk, and M. Sprangers. 2012. Do inflammatory bowel disease patients with anxiety and depressive symptoms receive the care they need? J. Crohns Colitis 6:68-76. https://doi .org/10.1016/j.crohns.2011.07.006.

Bloom, S. M., V. N. Bijanki, G. M. Nava, L. Sun, N. P. Malvin, D. L. Donermeyer, W. M. Dunne Jr., P. M. Allen, and T. S. Stappenbeck. 2011. Commensal Bacteroides species induce colitis in hostgenotype-specific fashion in a mouse model of inflammatory bowel disease. Cell Host Microbe 9:390-403. https://doi.org/10.1016/j .chom.2011.04.009.

Cui, C., Y. Lu, Y. Yue, S. Wu, S. Wang, M. Yu, and Z. Sun. 2020. Camel milk regulates T-cell proliferation to alleviate dextran sodium sulphate-induced colitis in mice. Int. J. Food Sci. Technol. 55:1648-1660.

Chen, B., J. Luo, Y. Han, H. Du, J. Liu, W. He, J. Zhu, J. Xiao, J. Wang, Y. Cao, H. Xiao, and M. Song. 2021. Dietary tangeretin alleviated dextran sulfate sodium-induced colitis in mice via inhibiting inflammatory response, restoring intestinal barrier function, and modulating gut microbiota. J. Agric. Food Chem. 69:76637674. https://doi.org/10.1021/acs.jafc.1c03046.

Chen, C., Y. M. Shah, K. Morimura, K. W. Krausz, M. Miyazaki, T. A. Richardson, E. T. Morgan, J. M. Ntambi, J. R. Idle, and F. J. Gonzalez. 2008. Metabolomics reveals that hepatic stearoyl-CoA desaturase 1 downregulation exacerbates inflammation and acute colitis. Cell Metab. 7:135-147. https://doi.org/10.1016/j.cmet .2007.12.003.

Dinan, T. G., and J. F. Cryan. 2017. Microbes, immunity, and behavior: psychoneuroimmunology meets the microbiome. Neuropsychopharmacology 42:178-192. https://doi.org/10.1038/npp.2016.103.

Du, B., F. Zhu, and B. Xu. 2018. An insight into the anti-inflammatory properties of edible and medicinal mushrooms. J. Funct. Foods 47:334-342. https://doi.org/10.1016/j.jff.2018.06.003.

Frank, D. N., A. L. St. Amand, R. A. Feldman, E. C. Boedeker, N. Harpaz, and N. R. Pace. 2007. Molecular-phylogenetic characterization of microbial community imbalances in human inflammatory bowel diseases. Proc. Natl. Acad. Sci. USA 104:13780-13785. https://doi.org/10.1073/pnas.0706625104.

Gevers, D., S. Kugathasan, L. A. Denson, Y. Vázquez-Baeza, W. Van Treuren, B. Ren, E. Schwager, D. Knights, S. J. Song, M. Yassour, X. C. Morgan, A. D. Kostic, C. Luo, A. González, D. McDonald, Y. Haberman, T. Walters, S. Baker, J. Rosh, M. Stephens, M. Heyman, J. Markowitz, R. Baldassano, A. Griffiths, F. Sylvester, D. Mack, S. Kim, W. Crandall, J. Hyams, C. Huttenhower, R. Knight, and R. J. Xavier. 2014. The treatment-naive microbiome in new-onset Crohn's disease. Cell Host Microbe 15:382-392. https: //doi.org/10.1016/j.chom.2014.02.005

Han, Y., M. Song, M. Gu, D. Ren, X. Zhu, X. Cao, F. Li, W. Wang, X. Cai, B. Yuan, T. Goulette, G. Zhang, and H. Xiao. 2019. Dietary intake of whole strawberry inhibited colonic inflammation in dextran-sulfate-sodium-treated mice via restoring immune homeostasis and alleviating gut microbiota dysbiosis. J. Agric. Food Chem. 67:9168-9177. https://doi.org/10.1021/acs.jafc.8b05581.

Huang, K., W. Dong, W. Liu, Y. Yan, P. Wan, Y. Peng, Y. Xu, X. Zeng, and Y. Cao. 2019. 2-O- $\beta$-D-Glucopyranosyl-L-ascorbic acid, an ascorbic acid derivative isolated from the fruits of Lycium barbarum L., modulates gut microbiota and palliates colitis in dextran sodium sulfate-induced colitis in mice. J. Agric. Food Chem. 67:11408-11419. https://doi.org/10.1021/acs.jafc.9b04411.

Jacobson, A. N., B. P. Choudhury, and M. A. Fischbach. 2018. The biosynthesis of lipooligosaccharide from Bacteroides thetaiotaomicron. MBio 9:e02289-e02217. https://doi.org/10.1128/mBio.02289 -17 .

Lagkouvardos, I., T. R. Lesker, T. C. Hitch, E. J. Gálvez, N. Smit, K. Neuhaus, J. Wang, J. F. Baines, B. Abt, B. Stecher, J. Overmann, 
T. Strowig, and T. Clavel. 2019. Sequence and cultivation study of Muribaculaceae reveals novel species, host preference, and functional potential of this yet undescribed family. Microbiome 7:28. https://doi.org/10.1186/s40168-019-0637-2.

Lavelle, A., and H. Sokol. 2020. Gut microbiota-derived metabolites as key actors in inflammatory bowel disease. Nat. Rev. Gastroenterol. Hepatol. 17:223-237. https://doi.org/10.1038/s41575-019-0258-z.

Liang, J., S. Chen, Y. Hu, Y. Yang, J. Yuan, Y. Wu, S. Li, J. Lin, L. He, S. Hou, L. Zhou, and S. Huang. 2018. Protective roles and mechanisms of Dendrobium officinal polysaccharides on secondary liver injury in acute colitis. Int. J. Biol. Macromol. 107:2201-2210. https://doi.org/10.1016/j.ijbiomac.2017.10.085.

Lin, J. C., J. Q. Wu, F. Wang, F. Y. Tang, J. Sun, B. Xu, M. Jiang, Y. Chu, D. Chen, X. Li, S. Su, Y. Zhang, N. Wu, S. Yang, K. Wu, and J. Liang. 2019. QingBai decoction regulates intestinal permeability of dextran sulphate sodium-induced colitis through the modulation of notch and NF- $\kappa B$ signalling. Cell Prolif. 52:e12547. https:/ /doi.org/10.1111/cpr.12547.

Maslowski, K. M., A. T. Vieira, A. Ng, J. Kranich, F. Sierro, H. C. Di Yu, M. S. Schilter, F. Rolph, D. Mackay, R. J. Artis, M. M. Xavier, Teixeira, and C. R. Mackay. 2009. Regulation of inflammatory responses by gut microbiota and chemoattractant receptor GPR43. Nature 461:1282-1286. https://doi.org/10.1038/nature08530.

Pei, R., D. A. Martin, J. C. Valdez, J. Liu, R. L. Kerby, F. E. Rey, J. A. Smyth, Z. Liu, and B. W. Bolling. 2019. Dietary prevention of colitis by aronia berry is mediated through increased Th17 and Treg. Mol. Nutr. Food Res. 63:e1800985. https://doi.org/10.1002/ mnfr.201800985.

Peng, L., X. Gao, L. Nie, J. Xie, T. Dai, C. Shi, L. Tao, Y. Wang, Y. Tian, and J. Sheng. 2020. Astragalin attenuates dextran sulfate sodium (DSS)-Induced acute experimental colitis by alleviating gut microbiota dysbiosis and inhibiting NF- $\mathrm{kB}$ activation in mice. Front. Immunol. 11:2058. https://doi.org/10.3389/fimmu.2020 .02058 .

Peng, Y., Y. Yan, P. Wan, D. Chen, Y. Ding, L. Ran, J. Mi, L. Lu, Z. Zhang, X. Li, X. Zeng, and Y. Cao. 2019. Gut microbiota modulation and anti-inflammatory properties of anthocyanins from the fruits of Lycium ruthenicum Murray in dextran sodium sulfateinduced colitis in mice. Free Radic. Biol. Med. 136:96-108. https:/ /doi.org/10.1016/j.freeradbiomed.2019.04.005.

Ryan, F. J., A. Ahern, R. Fitzgerald, E. Laserna-Mendieta, E. Power, A. Clooney, K. O'Donoghue, P. McMurdie, S. Iwai, A. Crits-Christoph, D. Sheehan, C. Moran, B. Flemer, A. L. Zomer, A. Fanning, J. O'Callaghan, J. Walton, A. Temko, W. Stack, L. Jackson, S. A. Joyce, S. Melgar, T. Z. DeSantis, J. T. Bell, F. Shanahan, and M. J. Claesson. 2020. Colonic microbiota is associated with inflammation and host epigenomic alterations in inflammatory bowel disease. Nat. Commun. 11:1512. https://doi.org/10.1038/s41467 -020-15342-5.

Schoultz, I., and Å. Keita. 2019. Cellular and molecular therapeutic targets in inflammatory bowel disease - Focusing on intestinal barrier function. Cells 8:193. https://doi.org/10.3390/cells8020193.
Shao, X., C. Sun, X. Tang, X. Zhang, D. Han, S. Liang, R. Qu, X. Hui, Y. Shan, L. Hu, H. Fang, H. Zhang, X. Wu, and C. Chen. 2020. Anti-inflammatory and intestinal microbiota modulation properties of Jinxiang garlic (Allium sativum L.) polysaccharides toward dextran sodium sulfate-induced colitis. J. Agric. Food Chem. 68:12295-12309. https://doi.org/10.1021/acs.jafc.0c04773.

Shi, J., Q. Xie, Y. Yue, Q. Chen, L. Zhao, S. E. Evivie, B. Li, and G. Huo. 2021. Gut microbiota modulation and anti-inflammatory properties of mixed lactobacilli in dextran sodium sulfate-induced colitis in mice. Food Funct. 12:5130-5143. https://doi.org/10 $.1039 / \mathrm{D} 1 \mathrm{FO} 00317 \mathrm{H}$.

Shu, Z., B. Yang, H. Zhao, B. Xu, W. Jiao, Q. Wang, Z. Wang, and H. Kuang. 2014. Tangeretin exerts anti-neuroinflammatory effects via NF- $\kappa$ B modulation in lipopolysaccharide-stimulated microglial cells. Int. Immunopharmacol. 19:275-282. https://doi.org/10 .1016/j.intimp.2014.01.011.

Su, L., L. Shen, D. R. Clayburgh, S. C. Nalle, E. A. Sullivan, J. B. Meddings, C. Abraham, and J. R. Turner. 2009. Targeted epithelial tight junction dysfunction causes immune activation and contributes to development of experimental colitis. Gastroenterology 136:551-563. https://doi.org/10.1053/j.gastro.2008.10.081.

Sun, J., H. Chen, J. Kan, Y. Gou, J. Liu, X. Zhang, X. Wu, S. Tang, R. Sun, C. Qian, N. Zhang, F. Niu, and C. Jin. 2020. Anti-inflammatory properties and gut microbiota modulation of an alkalisoluble polysaccharide from purple sweet potato in DSS-induced colitis mice. Int. J. Biol. Macromol. 153:708-722. https://doi.org/ 10.1016/j.ijbiomac.2020.03.053.

Suzuki, T., S. Yoshida, and H. Hara. 2008. Physiological concentrations of short-chain fatty acids immediately suppress colonic epithelial permeability. Br. J. Nutr. 100:297-305. https://doi.org/10 $.1017 /$ S0007114508888733.

Vinolo, M. A., H. G. Rodrigues, R. T. Nachbar, and R. Curi. 2011. Regulation of inflammation by short chain fatty acids. Nutrients 3:858-876. https://doi.org/10.3390/nu3100858.

Wang, K., Z. Wan, A. Ou, X. Liang, X. Guo, Z. Zhang, L. Wu, and X. Xue. 2019. Monofloral honey from a medical plant, Prunella vulgaris, protected against dextran sulfate sodium-induced ulcerative colitis via modulating gut microbial populations in rats. Food Funct. 10:3828-3838. https://doi.org/10.1039/C9FO00460B.

Zeng, Q., X. He, S. Puthiyakunnon, H. Xiao, Z. Gong, S. Boddu, L. Chen, H. Tian, S.-H. Huang, and H. Cao. 2017. Probiotic mixture Golden Bifido prevents neonatal Escherichia coli K1 translocation via enhancing intestinal defense. Front. Microbiol. 8:1798. https:/ /doi.org/10.3389/fmicb.2017.01798.

Zhang, H., Y. Wang, Y. Su, X. Fang, and W. Guo. 2021. The alleviating effect and mechanism of Bilobalide on ulcerative colitis. Food Funct. 12:6226-6239. https://doi.org/10.1039/D1FO01266E. 